\title{
QSAR modeling for acute toxicity prediction in rat by common painkiller drugs
}

\author{
Jinia Sinha Roy ${ }^{1 \mathrm{a}}$, Kaushik Gupta ${ }^{2 \mathrm{~b}}$ and Soumendra Nath Talapatra ${ }^{3 \mathrm{c}^{*}}$ \\ ${ }^{1}$ Career Advancement Solutions, H2 - 120A/ New, Benir Pole Road \\ Maheshtala, Kolkata - 700141, India \\ ${ }^{2}$ Career Advancement Solutions, H2 - 120A/ New, Benir Pole Road \\ Maheshtala, Kolkata - 700141, India \\ ${ }^{3}$ Career Advancement Solutions, H2 - 120A/ New, Benir Pole Road \\ Maheshtala, Kolkata - 700141, India \\ ajiniasinharoy2609@gmail.com, bkaushik.gupta101@gmail.com, cecologylive@yahoo.co.in \\ ${ }^{*}$ Corresponding Author \\ ${ }^{*}$ Corresponding author: Dr. Soumendra Nath Talapatra, Chief Scientist, H2 - 120A/New, Benir \\ Pole Road, Kolkata 700141. phone: +919831008689, email: ecologylive@yahoo.co.in
}

Keywords: QSAR; Mathematical modeling; Acute toxicity; Rat oral $\mathrm{LD}_{50}$; Common painkiller drugs

\begin{abstract}
Painkiller drugs or analgesics are potent pain reliever chemical agents, which are commonly used in pain therapy. Mathematical modeling by QSAR (quantitative structure activity relationship) methods are well known practices to determine predictive toxicity in biota. Now-adays, an easy screening of chemicals, QSAR can be done by using several recommended softwares. The present study was carried out by using software namely T.E.S.T. (Toxicity estimation software tool) for rat oral $\mathrm{LD}_{50}$ (median lethal dose) predictive toxicity for common painkiller drugs. These painkiller drugs were selected as 35 compounds and tabulated on the basis characteristics of one non-narcotic viz. acetaminophen, twenty non-steroidal anti-inflammatory such as bromofenac, diclofenac, diflunsial, etodolac, fenoprofen, flurbiprofen, ibuprofen, indomethacin, ketoprofen, ketorolac, maclofenamate sodium, mefenamic acid, meloxicam, nabumetone, naproxen, oxaprozin, phenylbutazone, piroxicam, sulindac and tolmetin as well as fourteen narcotic viz. buprenorphine, butorphanol, codeine, hydrocodone, hydromorphone, levorphanol, meperidine, methadone, morphine, nalbuphine, oxycodone, pentazocine, dextropropoxyphene and tapentadol. The data were tabulated on experimental (bioassay) from ChemIDPlus and T.E.S.T. and predictive toxicity of 30 compounds out of 35 compounds by using T.E.S.T. The predictive data were found by T.E.S.T. that 20 and 10 compounds were very toxic and moderately toxic respectively but not extremely, super toxic and non-toxic in rat model after acute oral exposure. It is suggested to evaluate the predicted data further with other available recommended softwares with different test models like daphnia, fish etc. to know aquatic toxicity when these compounds may discharge into waterbodies.
\end{abstract}

\section{INTRODUCTION}

From decades, the therapy of pain has been taken care by using medicines or drugs, these are known as analgesics. These analgesics are further subdivided into non-narcotic, narcotic and nonsteroidal anti-inflammatory drugs (NSAID). These drugs have capacity to relief pain but have potent side effects viz. gastrointestinal disorder, respiratory effect, renal toxicity etc. [1-2].

Quantitative structure-activity relationship (QSAR) is a model based on mathematics and can be done by using various softwares such as T.E.S.T. [3], TOPKAT (Toxicity Prediction by Komputer Assisted Technology) [4], DRAGON [5], ADMET (Absorption, Distribution, Metabolism, Elimination, and Toxicity) 2 and 3 [6] etc., which is related to the structure-derived features or molecular descriptors of particular chemical compound to detect its biological or physicochemical 
activity at an endpoint of $\mathrm{IC}_{50}, \mathrm{LC}_{50} / \mathrm{LD}_{50}, \mathrm{EC}_{50}$ etc. on biota. Therefore, this method has been established for the predictive and ultimately diagnostic abilities prior to experimental bioassay. Generally in QSAR methods, the molecular descriptors are used on the basis of thermodynamic, steric and electronic parameters [7]. Furthermore, the QSAR models can easily be used to understand drug action, design new chemicals or drugs and screen libraries of different compounds [8-12]. The experimental measurement (bioassay) with animal models for compounds are difficult because to get proper inbreed species, more expensive and time-consuming. According to Kovalishyn et al. [13], the prediction of biological activity through QSAR along with statistical modeling is a suitable method. Among all these recommended softwares, T.E.S.T. is a noncommercial, easy operation, based on 2D molecular descriptors and can be predicted of various toxicity endpoints for different test models [12, 14-16].

In this present study an attempt has been made to predict acute toxicity of common painkiller drugs in the rat oral exposure for $\mathrm{LD}_{50}$ values through QSAR modeling software package. The study emphasizes existing $\mathrm{LD}_{50}$ values through bioassay as experimental compared with predicted $\mathrm{LD}_{50}$ values by using T.E.S.T. (Toxicity Estimation Software Tool) software for different chemicals used as analgesics.

\section{MATERIALS AND METHODS}

\subsection{Name of the selected compounds investigated as painkiller drugs}

There were commonly used 35 types of painkiller drugs selected as per well-known investigation and tabulated their CAS (Chemical Abstracts Services) no., which were taken from ChemIDplus [17] of USEPA. These painkiller drugs were selected and tabulated on the basis characteristics of one non-narcotic viz. acetaminophen, twenty non-steroidal anti-inflammatory such as bromofenac, diclofenac, diflunsial, etodolac, fenoprofen, flurbiprofen, ibuprofen, indomethacin, ketoprofen, ketorolac, maclofenamate sodium, mefenamic acid, meloxicam, nabumetone, naproxen, oxaprozin, phenylbutazone, piroxicam, sulindac and tolmetin as well as fourteen narcotic viz. buprenorphine, butorphanol, codeine, hydrocodone, hydromorphone, levorphanol, meperidine, methadone, morphine, nalbuphine, oxycodone, pentazocine, dextropropoxyphene and tapentadol.

\subsection{Name of the software used}

In present study, the software was used namely T.E.S.T Verson 4.1 [3].

\subsection{Meta data used for $\mathrm{LD}_{50}$ of rat oral exposure}

Meta data as experimental values $(\mathrm{mg} / \mathrm{kg})$ for rat oral $\mathrm{LD}_{50}$ values were obtained from PubChem (ChemIDplus) and converted to Log $\mathrm{LD}_{50}$ value for individual painkiller drugs.

\subsection{QSAR modeling by using T.E.S.T. software}

The QSAR modeling software package was used to estimate the $\mathrm{LD}_{50}$ values of different categories of painkiller drugs. The software was used here namely Toxicity Estimation Software Tool or T.E.S.T., Version 4.1, developed by US EPA [3]. The present software was developed on the basis of two-dimensional (2D) molecular descriptors. The acute toxicity prediction of rat oral $\mathrm{LD}_{50}$ values were compared between bioassay results as experimental value obtained from PubChem (ChemIDplus) and predicted values were obtained after calculating the above mentioned software by incorporating CAS no. of individual chemical. Hence, CAS no. is an important input to predict acute toxicity in this particular modeling software.

According to Martin et al. [18], the T.E.S.T. software package estimates toxicity using a variety of QSAR methodologies namely hierarchical clustering, the Food and Drug Administration (FDA) 
MDL, nearest neighbour and a consensus model. In the present study, the data obtained from consensus method was only tabulated in the predicted toxicity results because the value is of average data by hierarchical clustering, the Food and Drug Administration (FDA) MDL, nearest neighbour methods. The structure of an individual chemical was visualized after entering CAS no and obtained a message that molecule ID successfully imported from the database of validated structures by software itself. Now the last step was, clicked the calculate option for the acute toxicity endpoint $\left(\mathrm{LD}_{50}\right)$ of rat by oral exposure of particular chemical in the present software.

The method for software operation and predictive $\mathrm{LD}_{50}$ data obtained as per instruction manual for the present software [3]. The statistical interpretation data as linear regression graph with $\mathrm{R}^{2}$ value in relation to prediction was obtained from PredictionResultsFDACluster.docx.

\section{RESULTS}

In Table 1, the acute toxicity prediction data were tabulated, which were obtained by using T.E.S.T. software. Out of the 35 selected painkiller drugs, the experimental bioassay as rat oral $\mathrm{LD}_{50}$ values $(\mathrm{mg} / \mathrm{kg})$ of 23 drugs viz. acetaminophen (1943.10), diclofenac (62.45), diflunsial (392.02), etodolac (94.07), flurbiprofen (116.92), ibuprofen (636.09), indomethacin (2.42), ketoprofen (62.42), mefenamic acid (740.59), nabumetone (3877.26), naproxen (247.89), oxaprozin (4470.66), phenylbutazone (244.98), piroxicam (215.94), sulindac (264.23), tolmetin (292.72), codeine (426.83), levorphanol (150.18), meperidine (161.94), methadone (86.03), morphine (335.28), pentazocine (1110.61) and dextropropoxyphene (135.17) were only reported in PubChem (ChemIDplus) database while bioassay data of twelve drugs viz. bromofenac, fenopeofen, ketorolac, maclofenamate sodium, meloxicam, buprenorphine, butorphanol, hydrocodone, hydromorphone, nalbuphine, oxycodone and tapentadol were not found in ChemIDPlus database.

In case of predictive toxicity study in rat orally exposed painkiller drugs, it was obtained the exact predicted $\mathrm{LD}_{50}$ values for 30 compounds but the T.E.S.T. unable to calculate 5 compounds due to unidentified CAS No. in the software. Out of 30 compounds, 6 compounds were predicted by T.E.S.T. but not considered in residual data because of unavailability of bioassay data. The prediction of $\mathrm{LD}_{50}$ values of rat oral exposure were obtained for 30 compounds viz. acetaminophen (1806.98), diclofenac (244.02), diflunsial (512.69), etodolac (764.60), flurbiprofen (762.47), ibuprofen (1713.58), indomethacin (228.66), ketoprofen (339.52), ketorolac (128.77), mefenamic acid (232.08), meloxicam (130.96), nabumetone (1613.76), naproxen (634.43), oxaprozin (733.40), phenylbutazone (972.11), piroxicam (1334.62), sulindac (473.82), tolmetin (229.76), butorphanol (699.85), codeine (204.84), hydrocodone (443.56), hydromorphone (323.17), levorphanol (227.31), meperidine (348.01), methadone (150.73), morphine (235.14), nalbuphine (398.56), oxycodone (443.66), pentazocine (391.90) and dextropropoxyphene (345.73) by using software T.E.S.T. consensus method (Table 1).

All the predicted and experimental $\mathrm{LD}_{50}$ values were calculated in $\log \mathrm{LD}_{50}$ values for 23 compounds. The $\mathrm{R}^{2}$ value of prediction data of 22 compounds from FDA cluster model fit results for individual compound was tabulated and the residual value of 23 compounds was also calculated in Table 2. It was noted that lower the residual value, least differences between experimental and predicted data. In T.E.S.T, the similarity analysis have found several compounds with very close similarity distance in the database. The confidence in the assessment between experimental and predicted of $\log \mathrm{LD}_{50}$ values $(\mathrm{mol} / \mathrm{kg})$ were represented graphically for individual chemical used as painkiller drug, which was calculated by software itself and all the graphs are exhibited in Fig. 1 and all the drugs were sub-numbered as a-v. 


\section{DISCUSSION}

Analgesic medicines are diverse groups of chemicals and established toxic impacts on biota [19]. The present prediction results were established that QSAR technique can be advantageous to detect relationship between the chemicals of non-narcotic, narcotic and NSAID molecular structures and their acute toxicities. In the present study, the T.E.S.T. software was estimated internally, the predicted rat oral $\mathrm{LD}_{50}$ values with the help of calculating inbuilt related $2 \mathrm{D}$ molecular descriptors. These molecular descriptors have already been calculated to detect mammalian predictive toxicity by QSAR modeling [12, 14, 16, 20-22]. For statistical interpretation to know the significant level $(>50 \%)$, linear regression analysis is a relevant method at the end of QSAR study [23], which has internally done by T.E.S.T itself.

In this present study, the $\mathrm{LD}_{50}$ predicted values were compared with the available experimental data of painkiller drugs as an individual chemical from ChemIDplus [17] for QSAR modeling by using T.E.S.T. software, which is supported by Ruiz et al. [14]. According to their previous studies, T.E.S.T. software has better capacity than other softwares like ADMET and TOPKAT for the prediction of sulphur mustard and its breakdown products on rat oral $\mathrm{LD}_{50}$ values. Canadian Center for Occupational Health \& Safety [24] and Ruiz et al. [14] have documented that the toxicity ranges as value of $<5 \mathrm{mg} / \mathrm{kg}, 5-50 \mathrm{mg} / \mathrm{kg}, 50-500 \mathrm{mg} / \mathrm{kg}, 500-5,000 \mathrm{mg} / \mathrm{kg}, 5,000-15,000 \mathrm{mg} / \mathrm{kg}$ and $>15,000 \mathrm{mg} / \mathrm{kg}$ is super toxic, extremely toxic, very toxic, moderately toxic, slightly toxic and practically non-toxic respectively have determined. The present predicted acute toxicity results with special reference to $\mathrm{LD}_{50}$ values of rat orally exposed to 20 types analgesics were obtained data for very toxic ranges compounds such as ketorolac $>$ meloxicam $>$ methadone $>$ codeine $>$ levorphanol $>$ indomethacin $>$ tolmetin $>$ mefenamic acid $>$ morphine $>$ diclofenac $>$ hydromorphone $>$ ketoprofen $>$ dextropropoxyphene $>$ meperidine $>$ pentazocine $>$ nalbuphine $>$ hydrocodone $>$ oxycodone $>$ sulindac $>$ diflunsial while 10 types moderately toxic compounds viz. naproxen $>$ butorphanol $>$ oxaprozin $>$ flurbiprofen $>$ etodolac $>$ phenylbutazone $>$ piroxicam $>$ nabumetone $>$ ibuprofen $>$ acetaminophen. The present results suggested to predicting toxicity with other aquatic test models viz. daphnia, fish etc. when these chemicals may get into the aquatic ecosystem with mixtures or individually through domestic wastewater [19].

\section{CONCLUSION}

It was concluded in the present work that the acute toxicity with special reference to rat oral $\mathrm{LD}_{50}$ value of painkiller drugs for experimental available data of ChemIDPlus [17] and predictive toxicity data through QSAR modeling software (T.E.S.T.) recommended by USEPA [3]. The results obtained for predicted acute toxicity data of twenty drugs viz. ketorolac, meloxicam, methadone, codeine, levorphanol, indomethacin, tolmetin, mefenamic acid, morphine, diclofenac, hydromorphone, ketoprofen, dextropropoxyphene, meperidine, pentazocine, nalbuphine, hydrocodone, oxycodone, sulindac and diflunsial were very toxic within range of $50-500 \mathrm{mg} / \mathrm{kg}$ while ten drugs viz. naproxen, butorphanol, oxaprozin, flurbiprofen, etodolac, phenylbutazone, piroxicam, nabumetone, ibuprofen and acetaminophen were moderately toxic within range of 500$5000 \mathrm{mg} / \mathrm{kg}$. This software helps to predict rat oral $\mathrm{LD}_{50}$ value with suitable programming of QSAR modeling based on 2D molecular descriptors and software calculates with similar test chemicals by evaluating test set and training set and the present study was evaluated the exact value of $\mathrm{LD}_{50}$ in rat exposed orally $[12,14]$. It was established that overdose of painkiller drugs cause severe toxicity [12]. Limited research work on QSAR modeling has been carried out on analgesics with other test species. This present prediction of painkiller drugs was based on only QSAR modeling software (T.E.S.T.) alongwith 2D molecular descriptors but should need further investigation by using other softwares of 3D molecular descriptors. It is suggested for future study, toxicity prediction should be done in other aquatic test models viz. daphnia, fish etc. with this software to know exact impact on non-mammals, when these compounds may found in wastewater and discharged into waterbodies. 
Table 1 - Prediction of $\mathbf{L D}_{50}$ values in rat by common painkiller drugs in comparison to available and unavailable bioassay metadata

\begin{tabular}{|c|c|c|c|c|c|c|c|c|}
\hline \multirow[t]{2}{*}{ SI no. } & \multirow[t]{2}{*}{ Generic name } & \multirow[t]{2}{*}{ CAS no.* } & \multicolumn{2}{|c|}{$\begin{array}{l}\text { Estimation by } \\
\text { bioassay } \\
\text { experiment }\end{array}$} & \multicolumn{2}{|c|}{$\begin{array}{l}\text { Software } \\
\text { estimation } \\
\text { T.E.S.T } \\
\text { (Consensus } \\
\text { method) }\end{array}$} & \multicolumn{2}{|c|}{$\begin{array}{lr}\text { Statistical } & \text { data } \\
\text { validation } & \text { by } \\
\text { T.E.S.T } & \end{array}$} \\
\hline & & & $\begin{array}{l}{ }^{*} \mathrm{LD}_{50} \\
\text { values } \\
(\mathrm{mg} / \\
\mathrm{kg})\end{array}$ & $\begin{array}{l}\text { Log } \\
\text { LD } \\
\text { values } \\
\text { (mg/ } \\
\text { kg) }\end{array}$ & $\begin{array}{l}\text { Predicted } \\
\text { LD }_{50} \\
\text { values } \\
(\mathrm{mg} / \\
\text { kg) }\end{array}$ & $\begin{array}{l}\text { Log } \\
\text { LD } \\
\text { value } \\
\text { (mg/ } \\
\text { kg) }\end{array}$ & $\begin{array}{l}\mathbf{R}^{2} \\
\text { value }^{\#}\end{array}$ & Residual \\
\hline 1. & Acetaminophen & $103-90-2$ & 1943.10 & 3.29 & 1806.98 & 3.26 & 0.85 & 0.03 \\
\hline 2. & Bromofenac & $91714-94-2$ & N.A. & --- & n.f. & --- & $\begin{array}{ll}--- \\
\end{array}$ & $\begin{array}{ll}--- \\
\end{array}$ \\
\hline 3. & Diclofenac & $15307-86-5$ & 62.45 & 1.79 & 244.02 & 2.39 & n.f. & -0.60 \\
\hline 4. & Diflunsial & $22494-42-4$ & 392.02 & 2.59 & 512.69 & 2.71 & 0.84 & -0.12 \\
\hline 5. & Etodolac & $41340-25-4$ & 94.07 & 1.97 & 764.60 & 2.88 & 0.83 & -0.91 \\
\hline 6. & Fenopeofen & $29679-58-1$ & N.A. & --- & n.f. & --- & --- & --- \\
\hline 7. & Flurbiprofen & 5104-49-4 & 116.92 & 2.07 & 762.47 & 2.88 & 0.88 & -0.81 \\
\hline 8. & Ibuprofen & $15687-27-1$ & 636.09 & 2.80 & 1713.58 & 3.23 & 0.83 & -0.43 \\
\hline 9. & Indomethacin & 53-86-1 & 2.42 & 0.38 & 228.66 & 2.36 & 0.83 & -1.98 \\
\hline 10. & Ketoprofen & $22071-15-4$ & 62.42 & 1.79 & 339.52 & 2.53 & 0.74 & -0.74 \\
\hline 11. & Ketorolac & 74103-06-3 & N.A. & --- & 128.77 & 2.11 & --- & --- \\
\hline 12. & $\begin{array}{l}\text { Maclofenamate } \\
\text { sodium }\end{array}$ & $67254-91-5$ & N.A. & --- & n.f. & --- & --- & --- \\
\hline 13. & Mefenamic acid & $61-68-7$ & 740.59 & 2.87 & 232.08 & 2.36 & 0.75 & 0.51 \\
\hline 14. & Meloxicam & $71125-38-7$ & N.A. & --- & 130.96 & 2.12 & --- & --- \\
\hline 15. & Nabumetone & $42924-53-8$ & 3877.26 & 3.59 & 1613.76 & 3.21 & 0.77 & 0.38 \\
\hline 16. & Naproxen & $22204-53-1$ & 247.89 & 2.39 & 634.43 & 2.80 & 0.77 & -0.41 \\
\hline 17. & Oxaprozin & 21256-18-8 & 4470.66 & 3.65 & 733.40 & 2.86 & 0.89 & 0.79 \\
\hline 18. & Phenylbutazone & $50-33-9$ & 244.98 & 2.39 & 972.11 & 2.99 & 0.79 & -0.60 \\
\hline 19. & Piroxicam & $36322-90-4$ & 215.94 & 2.33 & 1334.62 & 3.12 & 0.90 & -0.79 \\
\hline 20. & Sulindac & $38194-50-2$ & 264.23 & 2.42 & 473.82 & 2.67 & 0.89 & -0.25 \\
\hline 21. & Tolmetin & $26171-23-3$ & 292.72 & 2.47 & 229.76 & 2.36 & 0.75 & 0.11 \\
\hline
\end{tabular}




\begin{tabular}{|c|c|c|c|c|c|c|c|c|}
\hline 22. & Buprenorphine & $52485-79-7$ & N.A. & --- & n.f. & --- & --- & --- \\
\hline 23. & Butorphanol & $42408-82-2$ & N.A. & --- & 699.85 & 2.84 & --- & --- \\
\hline 24. & Codeine & $76-57-3$ & 426.83 & 2.63 & 204.84 & 2.31 & 0.82 & 0.32 \\
\hline 25. & Hydrocodone & $125-29-1$ & N.A. & --- & 443.56 & 2.65 & --- & --- \\
\hline 26. & Hydromorphone & $466-99-9$ & N.A. & --- & 323.17 & 2.51 & --- & --- \\
\hline 27. & Levorphanol & $77-07-6$ & 150.18 & 2.18 & 227.31 & 2.36 & 0.65 & -0.18 \\
\hline 28. & Meperidine & $57-42-1$ & 161.94 & 2.21 & 348.01 & 2.54 & 0.85 & -0.33 \\
\hline 29. & Methadone & $76-99-3$ & 86.03 & 1.93 & 150.73 & 2.18 & 0.74 & -0.25 \\
\hline 30 . & Morphine & $57-27-2$ & 335.28 & 2.52 & 235.14 & 2.37 & 0.78 & 0.15 \\
\hline 31. & Nalbuphine & $20594-83-6$ & N.A. & --- & 398.56 & 2.60 & --- & --- \\
\hline 32. & Oxycodone & $76-42-6$ & N.A. & --- & 443.66 & 2.65 & --- & --- \\
\hline 33. & Pentazocine & $359-83-1$ & 1110.61 & 3.04 & 391.90 & 2.59 & 0.92 & 0.45 \\
\hline 34. & $\begin{array}{l}\text { Dextropropoxy- } \\
\text { phene }\end{array}$ & $469-62-5$ & 135.17 & 2.13 & 345.73 & 2.54 & 0.74 & -0.41 \\
\hline 35. & Tapentadol & $175591-09-0$ & N.A. & --- & n.f. & --- & --- & --- \\
\hline
\end{tabular}

*All experimental bioassay data obtained from ChemIDplus [17] and T.E.S.T. [3]; N.A. = Not available n.f. $=$ Not found in T.E.S.T.; --- $=$ Not done; ${ }^{*}$ highest value of individual predicted data from FDA model fit results obtained from T.E.S.T.

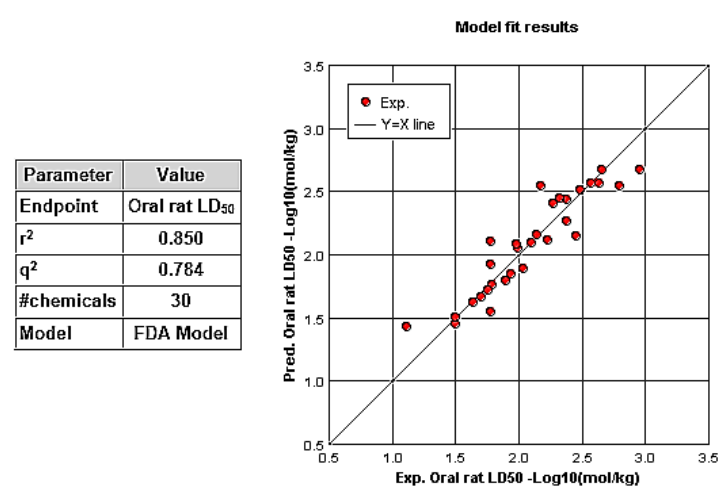

a. Acetaminophen
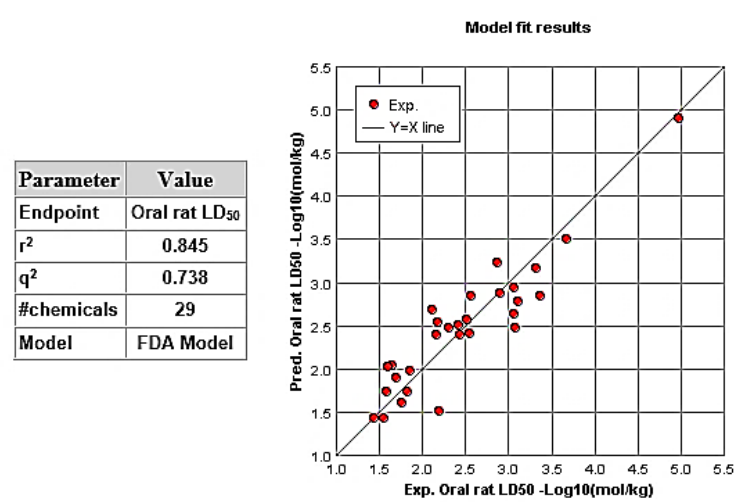

b. Diflunsial 


\begin{tabular}{|l|c|}
\hline Parameter & Value \\
\hline Endpoint & Oral rat $\mathrm{LD}_{50}$ \\
\hline $\mathbf{r}^{\mathbf{2}}$ & $\mathbf{0 . 8 2 6}$ \\
\hline $\mathbf{q}^{\mathbf{2}}$ & $\mathbf{0 . 7 1 9}$ \\
\hline$\#$ chemicals & $\mathbf{3 0}$ \\
\hline Model & FDA Model \\
\hline
\end{tabular}

c. Etodolac

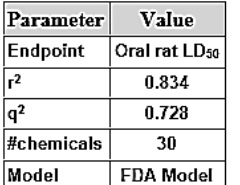

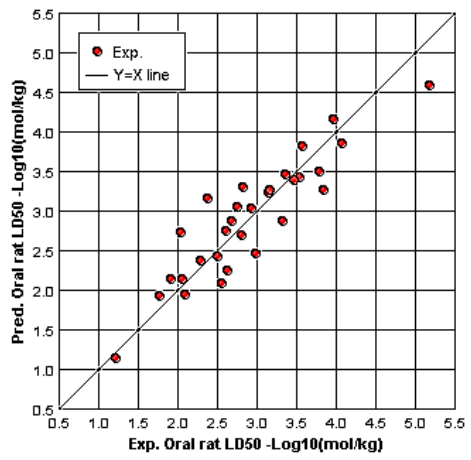

Parameter Value \begin{tabular}{|l|l|}
\hline Endpoint & Oral rat $\mathrm{LD}_{50}$ \\
\hline$r^{2}$
\end{tabular}

\begin{tabular}{|l|c|}
\hline $\mathrm{r}^{2}$ & 0.877 \\
\hline $\mathrm{q}^{2}$ & 0.821 \\
\hline$\#$ \#chemicals & $\mathbf{3 0}$ \\
\hline Model & FDA Model \\
\hline
\end{tabular}

\begin{tabular}{l|l}
\hline Model & FDA Model \\
\hline
\end{tabular}

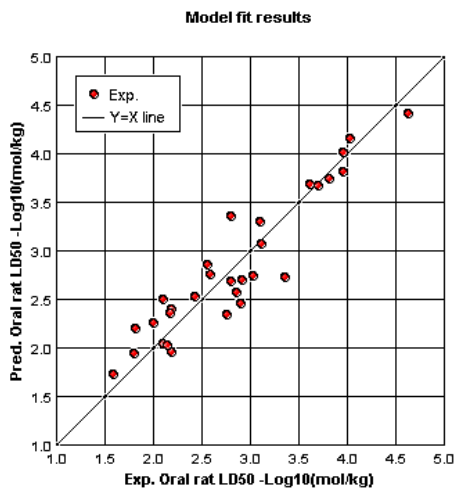

d. Flurbiprofen

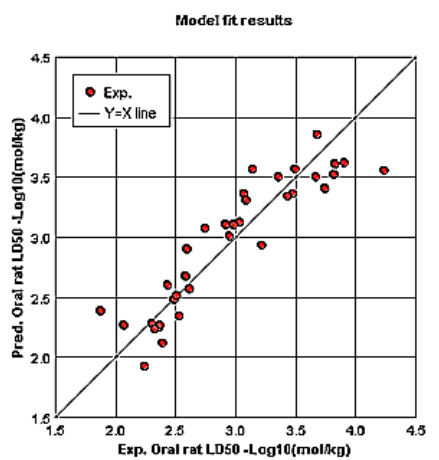

f. Indomethacin

Model fit results

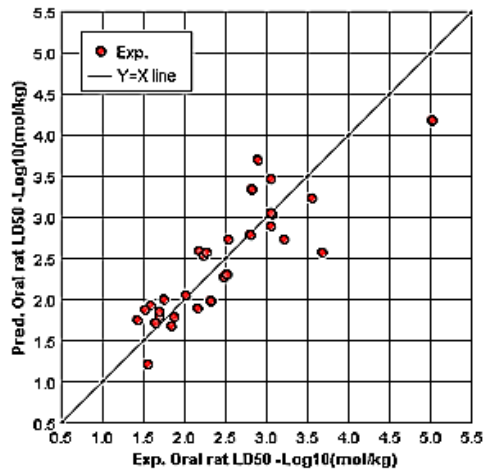

h. Mefenamic acid

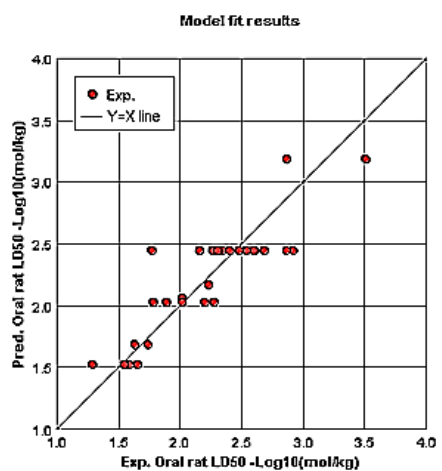

\section{g. Ketoprofen}

\begin{tabular}{|l|c|}
\hline Parameter & Value \\
\hline Endpoint & Oral rat $\mathrm{LD}_{50}$ \\
\hline $\mathbf{r}^{\mathbf{2}}$ & 0.773 \\
\hline $\mathbf{q}^{2}$ & 0.643 \\
\hline \#chemicals & 30 \\
\hline Model & FDA Model \\
\hline
\end{tabular}

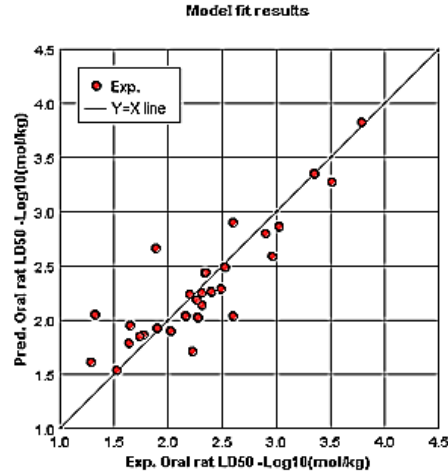

i. Nabumetone j. Naproxen

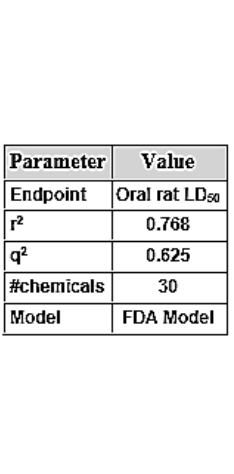




\begin{tabular}{|l|c|}
\hline Parameter & Value \\
\hline Endpoint & Oral rat LD $\mathrm{D}_{50}$ \\
\hline $\mathrm{r}^{2}$ & 0.887 \\
\hline $\mathrm{q}^{2}$ & 0.797 \\
\hline \#chemicals & 30 \\
\hline Model & FDA Model \\
\hline
\end{tabular}

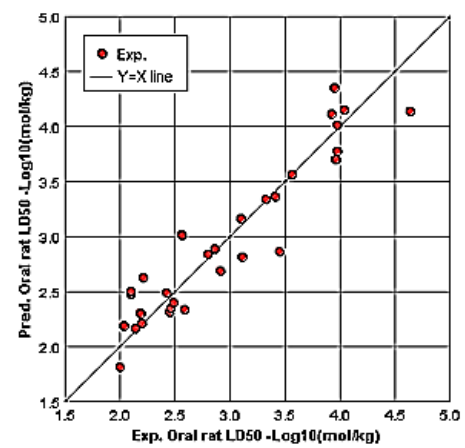

k. Oxaprozin

Model fit results

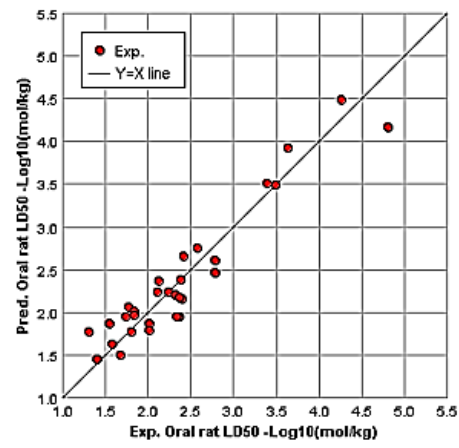

m. Piroxicam

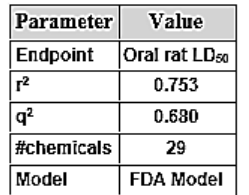

\begin{tabular}{|l|c|}
\hline Parameter & Value \\
\hline Endpoint & Oral rat $\mathrm{LD}_{50}$ \\
\hline $\mathrm{r}^{2}$ & 0.648 \\
\hline $\mathrm{q}^{2}$ & 0.504 \\
\hline \#chemicals & 35 \\
\hline Model & FDA Model \\
\hline
\end{tabular}

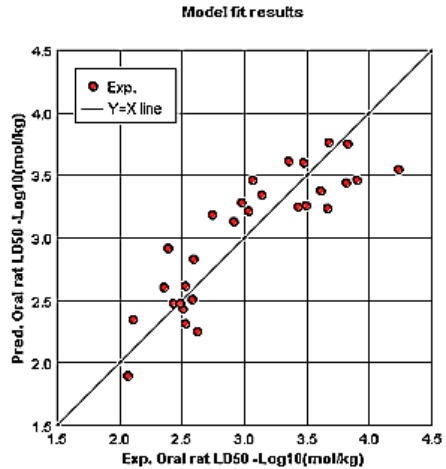

\section{o. Tolmetin}

Model if results

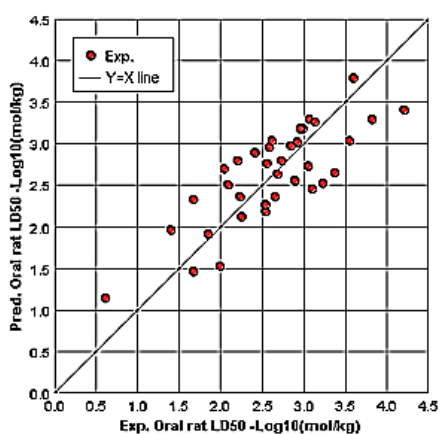

q. Levorphanol
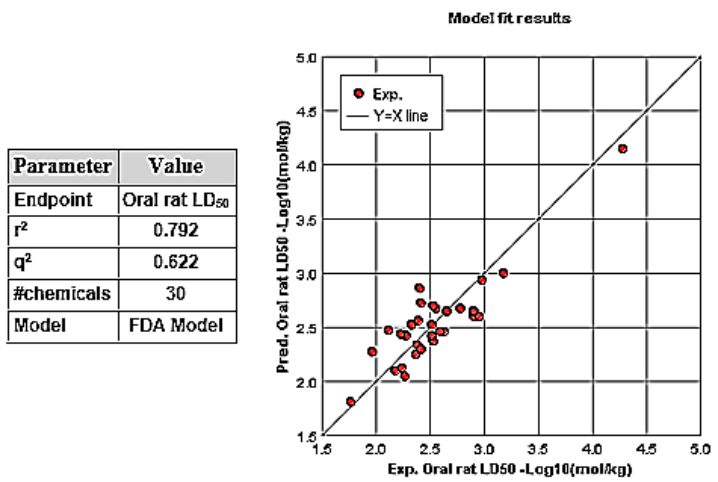

\section{Phenylbutazone}

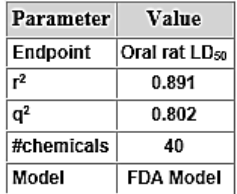

\begin{tabular}{|l|l|}
\hline Model & FDA Model \\
\hline
\end{tabular}

\begin{tabular}{|l|c|}
\hline Parameter & Value \\
\hline Endpoint & Oral rat $\mathrm{LD}_{s 0}$ \\
\hline $\mathrm{r}^{2}$ & 0.817 \\
\hline $\mathrm{q}^{2}$ & 0.700 \\
\hline ffchemicals & 30 \\
\hline Model & FDA Model \\
\hline
\end{tabular}

\begin{tabular}{|l|c|}
\hline Parameter & Value \\
\hline Endpoint & Oral rat $\mathrm{LD}_{s 0}$ \\
\hline $\mathrm{r}^{2}$ & 0.848 \\
\hline $\mathbf{q}^{2}$ & 0.714 \\
\hline \#chemicals & 30 \\
\hline Model & FDA Model \\
\hline
\end{tabular}

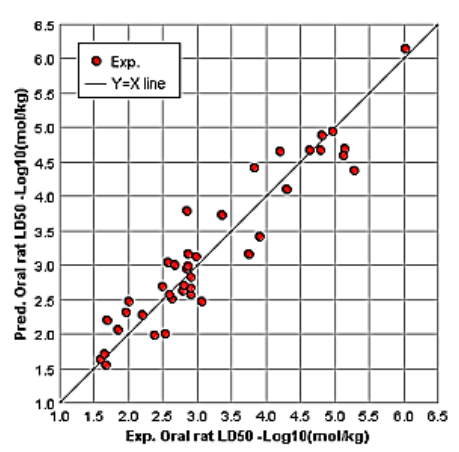

\section{n. Sulindac}

Model fit results

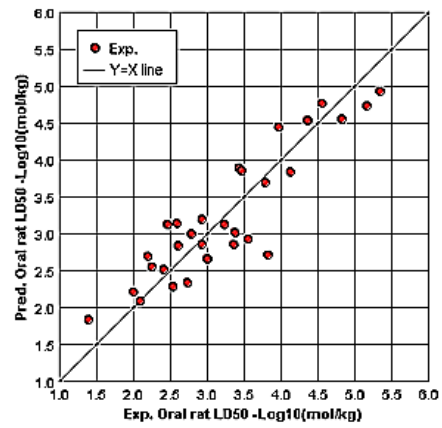

\section{p. Codeine}

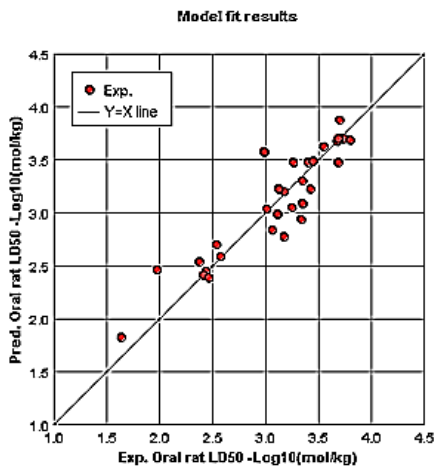

r. Meperidine 


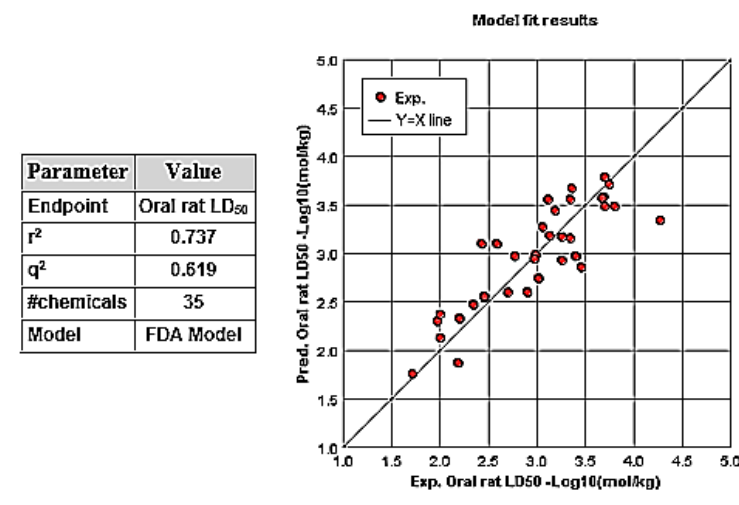

s. Methadone

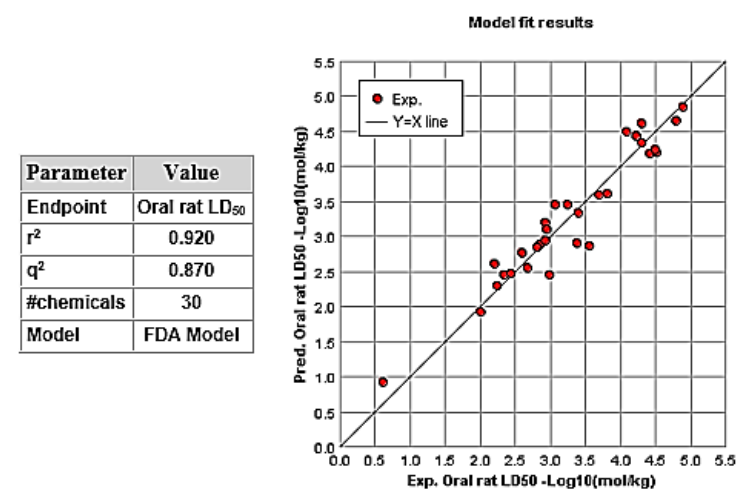

u. Pentazocine
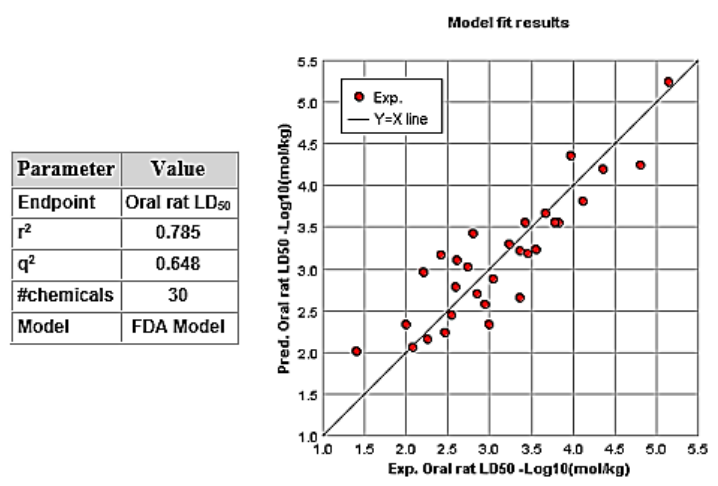

t. Morphine
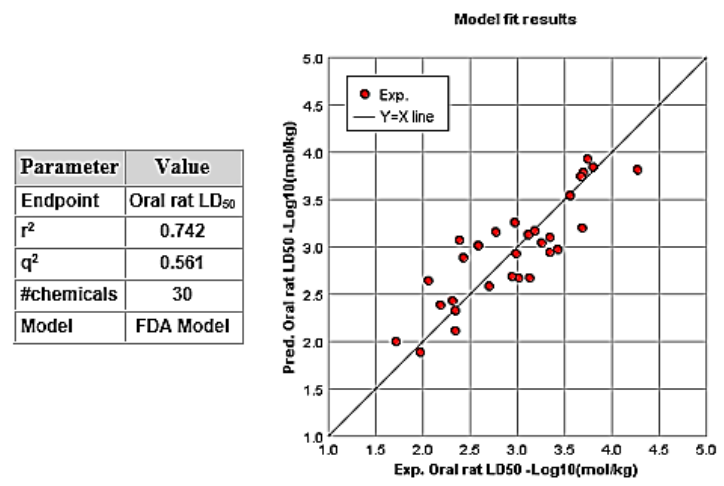

v. Dextropropoxyphene

Fig 1. Statistical analysis and graph for different painkiller drugs (a-v) prediction (data obtained from T.E.S.T. software)

\section{REFERENCES}

[1] W.O. Foye, Principles of Medicinal Chemistry, 3rd edition, Bombay: Varghese Publishing House, (1989) p. 240.

[2] S. Arora, and Saurabhvija, QSAR study on some newly synthesized pyrimido-benzimidazole derivatives as analgesic agents, Int. J. Pharm. Pharm. Sci. 3(5suppl), (2011) 457-461.

[3] USEPA (United States Environmental Protection Agency) T. E. S. T Tool, User's Guide for T.E.S.T, Version 4.1, A Program to Estimate Toxicity from Molecular Structure, Cincinnati, OH, USA, (2012).

[4] Accelrys Inc., TOPKAT software, Accelrys Inc., San Diego, CA, (2003).

[5] Talete, Dragon Version 5.4, (2006) (http://www.talete.mi.it/dragon_net.htm)

[6] User Manual ADMET, Version 5.5., Simulation Plus Inc, S.P.: Lancaster, CA, USA, (2011).

[7] F. Choplin, Comprehensive medicinal chemistry, Corwin Hansch, Vol 4, Elsevier Pergamon, Oxford, (2005) 33-57.

[8] C.W. Yap, Y. Xue, Z.R. Li, Y.Z. Chen, Application of support vector machines to in silico prediction of cytochrome P450 enzyme substrates and inhibitors, Curr. Topics Med. Chem. 6 (15) (2006) 1593-1607.

[9] R.V. Guido, G. Oliva, A.D. Andricopulo, Virtual screening and its integration with modern drug design technologies, Curr. Med. Chem. 15 (1) (2008) 37-46. 
[10] A. Schwaighofer, T. Schroeter, S. Mika, G. Blanchard, How wrong can we get? A review of machine learning approaches and error bars, Comb. Chem. High Throughput Screen. 12 (5) (2009) 453-468.

[11] L.G. Valerio Jr., In silico toxicology for the pharmaceutical sciences, Toxicol. Appl. Pharmacol. 241 (2009) 356-370.

[12] S.N. Talapatra, D. Misra, K. Banerjee, P. Banerjee, S. Swarnakar, QSAR modeling for acute toxicity prediction of fluroquinolone antibiotics by using software, Int. J. Adv. Res. 3 (6) (2015) 225-240.

[13] V. Kovalishyn, I. Kopernyk, S. Chumachenko, O. Shablykin, F. Kondratyuk, S. Pil'o, V. Prokopenko, V. Brovarets, L. Metelytsia, QSAR studies, design, synthesis and antimicrobial evaluation of azole derivatives, Comput. Biol. Bioinfor. 2(2) (2014) 25-32.

[14] P. Ruiz, G. Begluitti, T. Tincher, J. Wheeler, M. Mumtaz, Prediction of acute mammalian toxicity using QSAR methods: A case study of sulfur mustard and its breakdown products, Molecules 17 (2012) 8982-9001.

[15] P. Banerjee, S.N. Talapatra, Assessment of medicinal tree diversity in the Chintamoni Kar Bird Sanctuary (CKBS), Kolkata, India and prediction of antimutagenic phytochemicals by using software, Int. J. Adv. Res. 3 (7) (2015) 225-243.

[16] S.N. Talapatra, A. Sarkar, Acute toxicity prediction of synthetic and natural preservatives in rat by using QSAR modeling software, Int. J. Adv. Res. 3 (7) (2015) 1424-1438.

[17] ChemIDplus, A Toxnet Database, U.S. National Library of Medicine, Available from: www.chem.sis.nlm.nih.gov/chemidplus.

[18] T.M. Martin, P. Harten, R. Venkatapathy, S. Das, D.M. Young, A hierarchical clustering methodology for the estimation of toxicity, Toxicol. Mech. Methods 18 (2008) 251-266.

[19] M. Cleuvers, Mixture toxicity of the anti-inflammatory drugs diclofenac, ibuprofen, naproxen, and acetylsalicylic acid. Ecotoxicol. Environ. Saf. 59 (2004) 309-315.

[20] V.K. Gombar, D.V.S. Jain, Quantification of molecular shape and its correlation with physicochemical properties, Indian J. Chem. 24A (1987) 554-555.

[21] V.K. Gombar, K. Enslein, Quantitative structure-activity relationship (QSAR) studies using electronic descriptors calculated from topological and molecular orbital (MO) methods, QSAR 9 (1990) 321-325.

[22] L.H. Hall, B. Mohney, L.B. Kier, The electrotopological state: Structure information at the atomic level for molecular graphs, J. Chem. Inf. Comput. Sci. 31 (1991) 76-82.

[23] S.J. Xu, Computer-assisted drug molecular design, Chemical Industry Press, Beijing, China (2004).

[24] Canadian Center for Occupational Health \& Safety, What is an $\mathrm{LD}_{50}$ and $\mathrm{LC}_{50}$, (2012), Available from: http://www.ccohs.ca/oshanswers/chemicals/LD50.html\#_1_6. 